
A. Gildone
B. Punginelli
M. Manfredini
A. Artioli
R. Faccini

\section{A comparison of two rehabilitation protocols after total knee arthroplasty: does flexion affect mobility and blood loss?}

Received: 28 August 2006

Accepted: 16 January 2007

Published online: 5 March 2007

\author{
A. Gildone $(\varangle) \cdot$ M. Manfredini \\ A. Artioli • R. Faccini \\ Operative Unit of Orthopaedics \\ and Traumatology \\ Del Delta Hospital \\ Via Valle Oppio \\ I-40023 Lagosanto (FE), Italy \\ E-mail: anbote@yahoo.it \\ B. Punginelli \\ Department of Physiotherapy \\ Del Delta Hospital \\ Lagosanto (FE), Italy
}

\author{
Abstract We report a prospective \\ trial comparing the effectveness of a \\ post-operative flexion regime versus \\ a standard extension regime on the \\ early outcome and on the post-opera- \\ tive blood loss of total knee arthro- \\ plasty. Fourty-eight knees were \\ divided into two different post-opera- \\ tive rehabilitation regimes: a flexion \\ regime and an extension regime. The \\ two groups were well matched with \\ respect to age, gender, operation side \\ and pre-op diagnosis. All patients \\ were implanted with a NexGen \\ cemented total knee prosthesis and \\ all operations were performed by the \\ same surgeon. Patients were assessed \\ pre-operatively, at the time of dis- \\ charge, at 6 weeks and at 12 weeks, \\ and were evaluated by means of the \\ Knee Society Score (KSS) and the \\ WOMAC score, the Clarkson criteria \\ for range of motion and muscolar
}

strength measurement, and the Verbal Numeric Scale (VNS) for the pain. Futhermore, postoperative blood loss was assessed by comparing the volume of blood in the drain at the time of their removal and measuring the difference in preoperative and postoperative blood haemoglobin $(\mathrm{Hb})$ in the 2 groups. Patients subjected to the flexion regime had a better KSS and Womac score after 12 weeks and had less post-operative blood loss, requiring fewer blood transfusions. No differences were found between the two groups in terms of pain and muscolar strength. We believe a flexion regime after a total knee arthroplasty is a valid option of rehabilitation treatment and does not result in an increase in wound problems.

Key words Knee arthroplasty • Blood loss • Postoperative flexion

\section{Introduction}

Total knee arthroplasty (TKA) is one of the most popular and effective surgical treatments. The outcome of TKA can be affected by the design of the prosthesis, the materials used, the surgical technique employed, and the physical characteristics of the patient at the time of surgery. Another variable that should be considered is the rehabilitation protocol, as it influences both early and late range of movement (ROM), postoperative pain and time to discharge. In order to gain a better and quicker rehabilitation, continous passive motion (CPM) devices have been used together with physiotherapy after TKA for the past two decades [1]. Traditionally, CPM has been administered by moving the knee from full extension through increasing degrees of flexion [2]; this rehabilitation protocol has been shown to be safe and effective in early postoperative rehabilitation, as it increases early ROM [3-5], decreases the need for remanipulation [6] and decreases inpatient stay $[2,3]$. Moreover, it has not been shown to compromise wound healing $[3,7]$ or increase fixed flexion deformity [3]; despite this, CPM is not perfect. Pope et al. [8] showed that CPM increases blood loss and analgesic needs.

In the last decade, an early flexion regime has been advocated in which the knee is progressively moved from 
flexion to extension [9-12], offering the benefits of early flexion to $90^{\circ}$. Furthermore, recently Sharma [13] showed that this protocol affords a lower post-operative blood loss, with lesser need of transfusions, which are costly and can give transfusion-associated infections. Jordan et al. [10] conducted a nonrandomized trial comparing standard and early flexion CPM after primary TKA. Early flexion resulted in decreased length of stay (LOS), decreased hospital costs, and increased ROM at 1 year. On the other hand, MacDonald et al. [11] found no significant differences, at any measured interval, among standard and early flexion CPM regimes and a no-CPM control group.

Therefore, the goal of this study was to test the hypothesis that flexion of the knee in the immediate postoperative period allows a better ROM and outcome and decreases postoperative blood loss and therefore the number of transfusions.

\section{Materials and methods}

Between October 2004 and November 2005, we performed a prospective trial of 48 patients undergoing primary unilateral knee arthroplasty due to severe osteoarthritis. The study included only patients admitted for primary knee arthroplasty; patients who had bleeding disorders or who were on anticoagulants in the preoperative period were excluded, as were patients who had previous knee surgery. We also excluded patients with body mass index (BMI) higher or equal to $33 \mathrm{~kg} / \mathrm{m}^{2}$, patients with severe diseases which could affect deambulation or bleeding (e.g. liver problems, rheumatoid arthritis, severe low back pain), and patients with severe heart or neurological diseases.

Surgical and rehabilitation protocols

In all cases, we implanted the NexGen total knee prosthesis (Zimmer, Warsaw, Indiana, USA) using standard technique, which consisted in a midline skin incision and a medial parapatellar arthrotomy, with patellar eversion; we never made patellar replacement. All operations were performed by the same surgeon (RF) at the same institution and under tourniquet control (250-350 $\mathrm{mmHg}$ ) after the limb was elevated or exsanguinated. The tourniquet was released after bandaging the limb at the end of the operation. In each knee, one intra-articular drain was used and removed the day after surgery. Capsule suturing was performed at $90^{\circ}$ of flexion, in both groups of patients. Analgesia was achieved in all patients by a PCA (patient-controlled analgesia) system using morphine, which was removed 48 hours after the operation and replaced by non-steroidal anti-inflammatory drugs (NSAIDs) if needed.

In all cases, we obtained axial radiographs of the patella 5 days after the operation, in order to let the patient flex the knee enough not to feel pain. Then we obtained standard radiographs (AP, lateral, axial) at 1 month, 3 months and 1 year. Patients also received $4000 \mathrm{IU}$ low molecular weight heparin (enoxaparin) for prophylaxis against deep vein thrombosis, started in the evening of the operative day, and they wore thromboembolic deterrent stockings in the postoperative period. Postoperative complications such as wound complications, thromboembolic complications or soft tissue hematomas were assessed. Clinically suspected thromboembolic complications were investigated with Doppler ultrasound or pulmonary scintigraphy.

Patients were assigned to either flexion or extension regime for postoperative mobilization. The flexion regimen was as follows:

- Day of operation. Following skin closure and wound dressings, the knee was placed on a $90^{\circ}$ splint, and held on this splint overnight.

- Day 1. During this day, pain control was mandatory; all patients still had a PCA system with morphine. Patients started the CPM with a ROM of $70^{\circ}-100^{\circ}$, twice a day for 2 hours each; the therapist moved the knee passively and actively and taught the patient to repeat these exercises during the day alone. Isometric strengthening of the quadriceps and concentric dynamic exercises of the periarticular hip and ankle muscles were encouraged. Overnight, the knee was again placed in the $90^{\circ}$ flexion splint.

- Day 2. Same as day 1, but the ROM allowed by the CPM device was $50^{\circ}-100^{\circ}$. After wearing thromboembolic deterrent stockings, the patient was allowed to sit on the bed or on a seat twice a day for 2 hours each and began knee mobilization exercises on the edge of the bed. Overnight, the knee was again placed in the $90^{\circ}$ flexion splint.

- Day 3. Same as day 2, but the ROM allowed by the CPM device was $0^{\circ}-100^{\circ}$. During the course of the day, the knee was alternatively kept in flexion with a foam wedge and then allowed to straighten out with a support under the ankle. The patient was allowed to sit on the bed or on a seat more than on day 2 and started to walk with a walker together with the therapist. Muscular strengthening exercises were increased.

- Day 4. Same as day 3, but the knee was kept free during the course of the day. The patient started to walk with two crutches.

The extension regimen was as follows:

- Day of operation. Following skin closure and wound dressing, the knee was placed straight on the bed for 24 hours, in an extension splint, with a pillow under the foot, in order to increase the venous backflow and decrease the edema. An ice bag over the knee allowed for the reduction of the hyperemia, the edema and the inflammation.

- Day 1. During the course of the day, CPM was applied twice for 2 hours each, in order to passively flex the knee with a ROM of $0^{\circ}-40^{\circ}$. The therapist passively and actively moved the knee and taught the patient to repeat these exercises during the day alone. Isometric strengthening of the quadriceps and concentric dynamic exercises of periarticular hip and ankle muscles were encouraged. Overnight, the knee was again placed in the extension splint.

- Day 2. Same as day 1, but the ROM allowed by the CPM device was $0^{\circ}-60^{\circ}$. After wearing thromboembolic deterrent stockings, the patient was allowed to sit on the bed or on a seat twice a day for 2 hours each and began knee mobilization exercises on the edge of the bed. 
- Day 3. Same as day 2, but the ROM allowed by the CPM device was $0^{\circ}-70^{\circ}$. The patient started to walk with a walker. Exercises for ROM and muscular strengthening recovery were intensified.

- Day 4. Same as day 3, but the ROM allowed by the CPM device was $0^{\circ}-90^{\circ}$; the patient started to walk with two crutches.

\section{Evaluation}

Postoperative blood loss was assessed by measuring the volume of blood in the drain at the time of removal. The postoperative hemoglobin $(\mathrm{Hb})$ drop was determined as the difference between preoperative and postoperative blood $\mathrm{Hb}$ concentrations. Postoperative hemoglobin was checked 48 hours after surgery. Blood transfusions were generally ordered when the postoperative $\mathrm{Hb}$ was less than $8 \mathrm{~g} / \mathrm{dl}$.

Functional assessment was performed pre-operatively, at the time of discharge, and after 6 and 12 weeks. Patients were evaluated by means of the Knee Society score (KSS) [14], the verbal numeric scale (VNS) for pain, the WOMAC score [15], and the Clarkson criteria for passive flexion and muscular strength measurement [16].

\section{Statistical analysis}

Statistical analysis included paired $t$ testing and multiple analysis of variance. A value of $p<0.05$ was considered significant.

\section{Results}

Of the 48 patients who underwent unilateral knee arthroplasty, 24 were assigned to a flexion regimen and the remaining 24 were assigned to an extension regimen of rehabilitation (Table 1). The groups were well matched with respect to age, gender, operation side and preoperative diagnosis.

The mean postoperative blood loss in the flexion regimen group was $302 \mathrm{ml}(\mathrm{SD}=110 \mathrm{ml})$, significantly less $(p=0.002)$ than that in the extension regimen group (mean, $495 \mathrm{ml}$; $\mathrm{SD}=83.5 \mathrm{ml}$ ) (Table 2). The postoperative $\mathrm{Hb}$ drop was also significantly higher in the knee extension group. There were three cases of wound dehiscence in the knee flexion group and none in the extension group. One patient in the knee flexion group received a blood transfusion compared to 4 patients in the knee extension group. In the extension group, patients with preoperative $\mathrm{Hb}$ concentrations less than 11.5 $\mathrm{g} / \mathrm{dl}$ were more likely to need a blood transfusion.

Table 1 Patients data

\begin{tabular}{lrr}
\hline & Flexion regime & Extension regime \\
\hline Numbers & 24 & 24 \\
Age & 73.2 (Range $61-88)$ & 72.7 (Range $66-84)$ \\
Males & 10 & 9 \\
Females & 14 & 15 \\
Operation side: right & 13 & 12 \\
Operation side: left & 11 & 12 \\
Osteoarthritis & 24 & 24 \\
\hline
\end{tabular}

Table 2 Pre- and post-operative means

\begin{tabular}{lrrr}
\hline Variable & Flexion regime mean (Range) & Extension regime mean (Range) & $p$ value \\
\hline Pre-operative KSS (clinical) & $55.125(36-73)$ & $61.583(40-78)$ & 0.02680239 \\
Pre-operative KSS (functional) & $54.583(20-80)$ & $58.958(5-80)$ & 0.285416791 \\
Post-operative KSS (6 wks) & $144.12(105-188)$ & $122.79(100-172)$ & 0.034122 \\
Post-operative KSS (12 wks) & $169.2(87-200)$ & $158.73(110-199)$ & 0.246589 \\
Pre-operative passive flexion & $115.41(105-120)$ & $113.14(110-125)$ & 0.5 \\
Post-operative passive flexion (6 wks) & $109.79(90-120)$ & $101.04(85-120)$ & 0.001003376 \\
Post-operative passive flexion (12 wks) & $116.66(110-120)$ & $110.83(100-125)$ & 0.005324572 \\
Pre-operative VNS & $6.21(4-9)$ & $6.92(5-9)$ & 0.067053983 \\
Post-operative VNS (6 wks) & $3.041(0-7)$ & $3.75(2-6)$ & 0.006528881 \\
Post-operative VNS (12 wks) & $1.33(0-3)$ & $2.25(2-7)$ & 0.001921419 \\
Pre-operative WOMAC & $72(65-80)$ & $73.29(36-109)$ & 0.71727556 \\
Post-operative WOMAC (12 wks) & $34.45(28-47)$ & $3.25(30-74)$ & 0.001194076 \\
Pre-operative flexor strength & $3.25(3-4)$ & $3.16(2-4)$ & 0.603642707 \\
Post-operative flexor strength (6 wks) & $3.41(2-4)$ & $3.91(3-4)$ & 0.203089172 \\
Post-operative flexor strength (12 wks) & $3.83(3-4)$ & $3.58(3-4)$ & 0.42587943 \\
Pre-operative extensor strength & $3.7(3-4)$ & $3.2(2-4)$ & 0.416990379 \\
Post-operative extensor strength (6 wks) & $3.66(3-4)$ & $3.91(3-4)$ & 0.095784 \\
Post-operative extensor strength (12 wks) & $4(4-4)$ & $495(360-620)$ & 0.082959876 \\
Post-operative blood loss (ml) & $301.66(150-520)$ & $2.81(1.5-3.7)$ & 0.000125 \\
Post-operative Hb drop (g/dl) & $2.31(1.2-3.4)$ & 0.0017 \\
\hline
\end{tabular}


The pre-operative KSS was divided in two parts: one regarding the outcome with regard to pain, ROM and stability, and one concerning the function of the operated knee. Mean pre-operative articular and functional KSS scores were lower in the flexion regimen patients when compared with the extension regimen patients, while the postoperative KSS of the first group were higher than those of the second group: this difference is clinically but not statistically significant. The VNS score for pain was similar between the two groups. Both groups presented a high mean score before surgery $(>6.0)$, which progressively lowered until week 12 . Differences in post-operative values are statistically and clinically significant, especially after 12 weeks. Before surgery, WOMAC scores of the two groups were similar and rather high, representing a sign of severe pain, stiffness and functional loss. After 12 weeks, the flexion group presented a lower mean WOMAC score than the extension group: this difference is both clinically and statistically significant. Regarding muscular strength, the groups presented similar trends in extensor and flexor muscle strength. In fact, before surgery patients' strength permitted anti-gravity movements, both in flexion and extension. Such strength was less at discharge because patients could not do antigravity movements but improved to normal values during the postoperative follow-up period. These differences are neither clinically nor statistically significant.

\section{Discussion}

Although pain relief is the most important determinant of the outcome of TKA, a good ROM is necessary for a satisfactory functional outcome. Jergeson et al. [17] showed that small changes in maximum flexion can have important effects on functional capability, while Ritter and Campbell [18] stated that a minimum flexion of $90^{\circ}$ is necessary for normal daily activities.

After continuous passive motion was introduced in 1976, patients started motion in the recovery room but the increase in flexion was slow because of concern for wound healing [7]; therefore, it usually took 10-18 days until patients were discharged with $85^{\circ}$ or more of flexion [7, 19-21]. The problem in restoring normal function after TKA has always been to obtain a consistent ROM irrespective of the large number of variables that can produce stiffness. We believe that an early flexion regimen, together with appropriate physiotherapy, can achieve this goal. Our results suggest that patients who had a flexion regimen of physiotherapy have better clinical and functional scores, better flexion at discharge, feel less pain at discharge and have better WOMAC scores. We did not find noticeable differences in muscular strength between the groups. Furthermore, we did not find problems regarding anterior knee pain in the flexion regimen group of patients during the follow-up period; in fact we found that, if they had to choose where to localize their pain, they would have chosen the posterior part of the knee, maybe because of a reflex contracture of the ischiocrural muscles and tendons.

Previously, Johnson raised concerns about flexing knees to $90^{\circ}$ in the early postoperative period, outlining that this regimen can have harmful effects on skin viability [7]. In fact, Ackroyd et al. [22] reported three cases of severe skin necrosis following a flexion regimen in which the knee was held predominantly in flexion for 5 days.

This study provides evidence that early flexion according to the reported protocol is safe. However, it should be noted that we took great care with wound closure in flexion and always ensured that dressings were not too constrictive. We believe that three nights with the knee held in flexion by a splint are enough to gain a good flexion; during the day, the knee is not fixed in flexion, but taken into progressive extension by the therapist and the CPM. Our flexion group had no increase in wound problems and there were no pressure problems as a result of lying with the leg flexed overnight. We have not seen skin necrosis either during the study or subsequently.

One of the main concerns about this kind of surgery is blood loss: we noticed that, when using CPM instead of complete extension, patients needed fewer blood transfusions. So, we believe that a flexion regimen can help patients restore better knee function and lose less blood as well. Although the exact mechanism of reduced blood loss in the flexion group remains unclear, we believe that a tamponade effect induced by reduced suprapatellar and patellofemoral spaces may be involved; another explanation is that the vessels of the popliteal fossa could be compressed when placed on a $90^{\circ}$ splint. However, we trust more in the tamponade effect of flexion over the suprapatellar pouch, due to the absence of complications caused by compression of the popliteal structures (e.g. phlebitis). In conclusion, maintaining the knee in $90^{\circ}$ flexion in the immediate postoperative period followed by continuous passive movement of the knee led to better outcomes at discharge and after 12 weeks in terms of passive flexion, KSS and WOMAC scores and blood loss, with a decrease in the need for transfusions, especially in patients with a pre-operative $\mathrm{Hb}$ concentration less than $12 \mathrm{~g} / \mathrm{dl}$. There were no substantial differences in terms of pain or muscular strength. 


\section{References}

1. Harms M, Engstrom B (1991) Continuous passive motion as an adjunct to treatment in the physiotherapy management of the total knee arthroplasty patient. Physiotherapy 77:301

2. Colwell CW, Morris BA (1992) The influence of continuous passive motion on the results of total knee arthroplasty. Clin Orthop 276:225-228

3. Johnson DP, Eastwood DM (1992) Beneficial effects of continuous vs extension suturing of total knee replacement wounds: a passive motion after total condylar arthroplasty. Ann R Coll Surg Engl 74:412-416

4. Shih KZ, Liu TK (1990) The role of continuous passive motion following total knee arthroplasty. J Formos Med Assoc 89:1077-1080

5. Romness DW, Rand JA (1996) The role of continuous passive total knee replacement. Clin Orthop 331:81-86

6. McInnes J, Larson MG, Daltoy LH et al (1992) A controlled evaluation of continuous passive motion in patients undergoing total knee arthroplasty. JAMA 268:1423-1428

7. Johnson DP (1990) The effect of continuous passive motion on wound healing and joint mobility after knee arthroplasty. J Bone Joint Surg Am 72:421-426

8. Pope RO, Corcoran S, McCaul K, Howie DW (1997) Continuous passive motion after primary total knee arthroplasty. Does it offer any benefits? J Bone Joint Surg Br 79:914-917
9. Ng TS, Yeo SJ (1999) An alternative early knee flexion regime of continuous passive motion for total knee arthroplasty. Physiotherapy Singapore 2:2

10. Jordan LR, Siegel JL, Olivo JL (1995) Early flexion routine: an alternative method of continuous passive motion. Clin Orthop Relat Res 315:231-233

11. MacDonald SJ, Bourne RB, Rorabeck $\mathrm{CH}$ et al (2000) Prospective randomized clinical trial of continuous passive motion after total knee arthroplasty. Clin Orthop Relat Res 380:30-35

12. Yashar AA, Venn-Watson E, Welsh T et al (1997) Continuous passive motion with accelerated flexion after total knee arthroplasty. Clin Orthop Relat Res 345:38-43

13. Sharma S (2004) Effects of knee flexion following knee arthroplasty on postoperative blood loss: a study on 50 patients. J Orthopaed Traumatol 2:86-91

14. Insall JN, Dorr LD, Scott RD, Scott WN (1989) Rationale of the Knee Society rating system. Clin Orthop 248:13-14

15. Bellamy N, Buchanan WW, Goldsmith $\mathrm{CH}$ et al (1988) Validation study of WOMAC: a health status instrument for measuring clinically important patient relevant outcomes to antirheumatic drug therapy in patients with osteoarthritis of the hip or knee. J Rheumatol 15:1833-1840
16. Clarkson HM (2002) Valutazione cinesiologica. Esame della mobilità articolare e della forza muscolare. Edi-Ermes, Milan

17. Jergeson HE, Poss R, Sledge CB (1978) Bilateral total hip and knee replacements in adults with rheumatoid arthritis: an evaluation of function. Clin Orthop 137:120-128

18. Ritter MA, Campbell ED (1987) Effect of range of motion on the success of a total knee arthroplasty. J Arthroplasty 2:95-97

19. Maloney WJ, Schurman DJ, Hangen D et al (1990) The influence of continuous passive motion on outcome in total knee arthroplasty. Clin Orthop 256:162-168

20. Vince KG, Kelly AM, Beck J, Insall JN (1987) Continuous passive motion after total knee arthroplasty. J Arthroplasty 2:281-284

21. Wasilewski SA, Woods LC, Torgerson Jr WR, Healy WL (1990) Value of continuous passive motion in total knee arthroplasty. Orthopedics 13:291-295

22. Ackroyd CE, Newman JH, Roberts H, Blazquez A (1998) Postoperative care after knee arthroplasty. Ann Orthopediques de L'Ouest 20:51-53 